\title{
Correlating Atomic-Scale Lattice and Nanoscale Structural Dynamics with Ultrafast Electron Microscopy
}

\author{
Spencer A. Reisbick ${ }^{1}$ and David J. Flannigan ${ }^{1 *}$ \\ 1. Department of Chemical Engineering and Materials Science, University of Minnesota, Minneapolis, \\ MN, USA. \\ * Corresponding author: flan0076@umn.edu
}

Ultrafast photoexcitation of transition metal dichalcogenides (TMDs) has been shown with ultrafast electron microscopy (UEM) imaging to produce coherent intra and interlayer low-frequency acoustic phonons that propagate along preferential wavevectors at the in-plane and $c$-axis speeds of sound [1-4]. In defect-laden crystals, coherent acoustic phonons were found to preferentially emerge from abrupt and extended crystal-crystal and crystal-vacuum interfaces. For certain morphologies and structural configurations, wave interference and phonon beating manifests as bend-contour motion that rapidly transitions from coherent to incoherent behaviors [3,4]. Nanosecond bright-field imaging over longer relaxation times revealed further relaxation of localized incoherent motion to lower-frequency, wholecrystal, coherent mechanical oscillations consisting of multiple modes having microsecond lifetimes. Owing to the highly anisotropic structures arising from in-plane covalent and $c$-axis van der Waals bonding, the elastic tensors and speeds of sound are significantly different along these crystallographic directions. With UEM, it was shown that the coherent $c$-axis acoustic-phonon dynamics could be monitored by tracking oscillatory moiré-fringe dynamics. Finite element modeling indicated fs photoexcitation initially produces an impulsive expansion along the layer stacking direction that rapidly couples to the in-plane propagating modes at discrete structural discontinuities, though direct evidence of this is currently lacking. Importantly, the nanoscale motions characteristic of these coherent structural dynamics must originate from electron-phonon coupling and atomic-scale variations in vibration amplitudes. Thus, reciprocal-space studies with UEM correlated with femtosecond brightfield imaging has the potential to provide a fuller picture of the overall structurally- and morphologically-dependent dynamics in these materials.

Here, we report the results of correlative ultrafast electron imaging and diffraction studies of the photoinduced structural dynamics of multi-layer $\mathrm{TaS}_{2}$. As with other TMDs studied with UEM, femtosecond photoexcitation of $\mathrm{TaS}_{2}$ produces highly coherent propagating acoustic phonons traveling along preferential wavevectors and seeded by morphological defect structures in the crystals. The structural dynamics manifest as coherent bend-contour motions, suggesting an oscillatory rocking of the reciprocal lattice with respect to a stationary Ewald sphere. Isolation of regions showing strong dynamics using selected-area apertures and monitoring responses in reciprocal space with ultrafast parallel-beam diffraction enables direct correlation of the real-space behaviors with the atomic-scale lattice dynamics through Bragg scattering. This selective lattice probing using apertures to isolate signal generated from sub-microscale specimen regions is especially useful for directly relating the observed dynamics to real-space motions, as the signals are in essence the same, the only difference being which plane - imaging or diffraction - is selected with the UEM lens settings. In this way, the coherent, propagating modes observed in real space could indeed be observed as Bragg-spot intensity oscillations in reciprocal space, with the individual frequencies being in strong agreement with one another. This reinforces the relrod/Ewald sphere picture, in that local lattice oscillations produce a spatial modulation of the scattering condition and a time-varying change in the excitation error without the need to invoke a 
Debye-Waller-type argument based on the structure factor. In addition to purely structural bend-contour dynamics, we also observe strongly-scattering regions associated with localized charge-density waves (CDWs) that behave in entirely distinct ways compared to the coherent acoustic phonons. The precise nature of the contrast in these regions appears to be directed by underlying defects, and modulation of the local morphologies in conjunction with lattice perturbations caused by the CDWs may contain the necessary information to quantitatively describe the observed real-space contrast dynamics [5].

References:

[1] DA Plemmons, PK Suri and DJ Flannigan, Chem. Mater. 27 (2015), p. 3178.

[2] DR Cremons, DA Plemmons and DJ Flannigan, Nat. Commun. 7 (2016), p. 11230.

[3] DR Cremons, DA Plemmons and DJ Flannigan, Struct. Dyn. 4 (2017), p. 044019.

[4] AJ McKenna, JK Eliason and DJ Flannigan, Nano Lett. 17 (2017), p. 3952.

[5] This work was supported partially by the National Science Foundation through the University of Minnesota MRSEC under Award Number DMR-1420013 and partially by the Arnold and Mabel Beckman Foundation in the form of a Beckman Young Investigator Award.
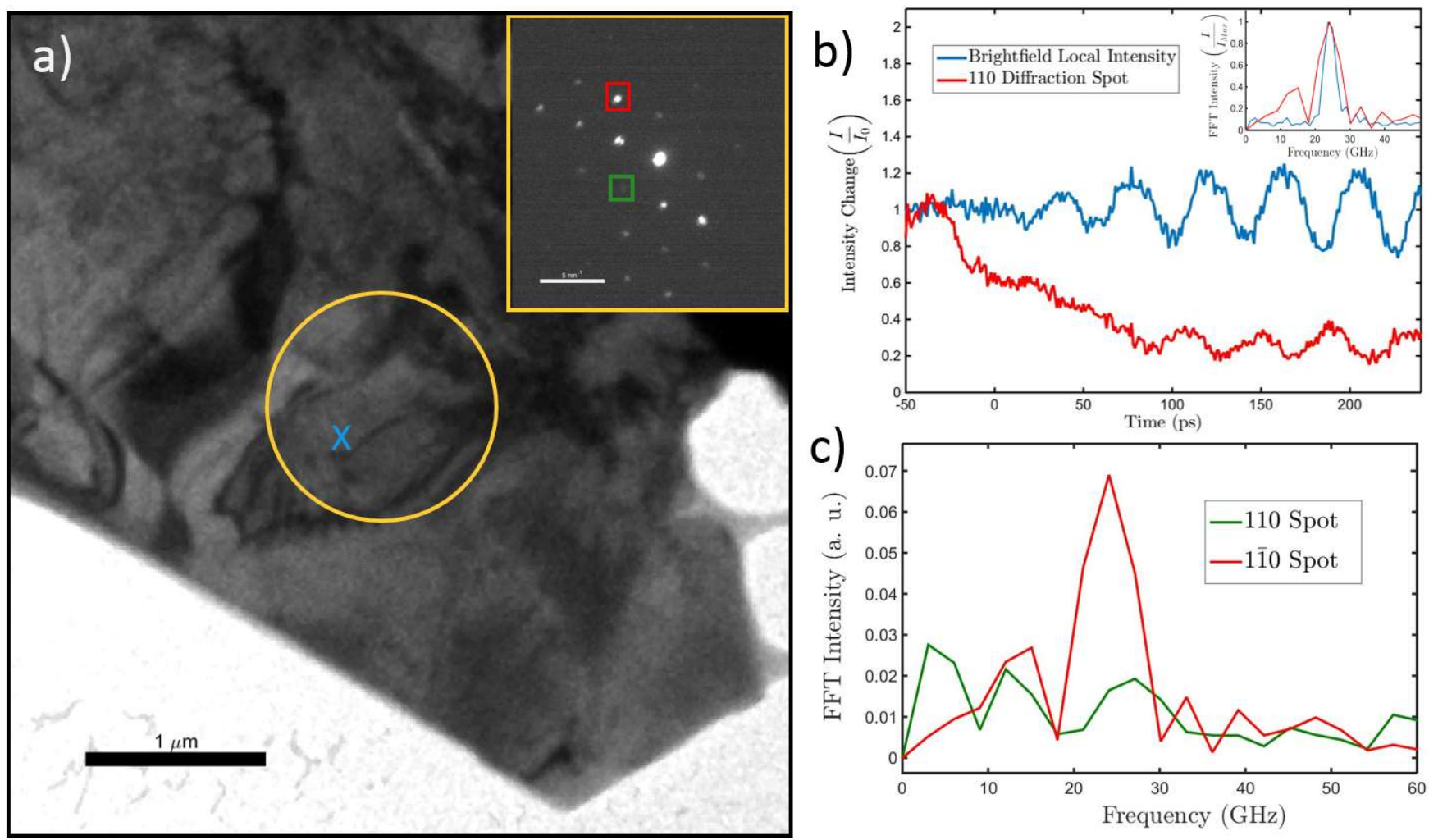

Figure 1. Correlative UEM imaging and diffraction of coherent acoustic-phonon dynamics in $1 \mathrm{~T}-\mathrm{TaS}_{2}$. (a) UEM bright-field image of a multi-layer $1 \mathrm{~T}-\mathrm{TaS}_{2}$ single crystal. The yellow circle denotes the position of the selected-area aperture used to conduct the UEM diffraction study. The blue X marks the position at which real-space dynamics were analyzed. The inset shows a representative UEM diffraction pattern. The Bragg spots highlighted are those analyzed and displayed in the subsequent panels. (b) Comparison of the 110 Bragg-spot dynamics (red) with the bright-field real-space dynamics (blue). The inset shows the FFTs of the time-domain trace. (c) FFTs of the time-domain dynamics of the Bragg spots highlighted in panel (a). 